\title{
Large serrated polyp with KRAS mutation in inflammatory bowel disease: a "nondysplastic dysplasia-associated lesion or mass (DALM)"?
}

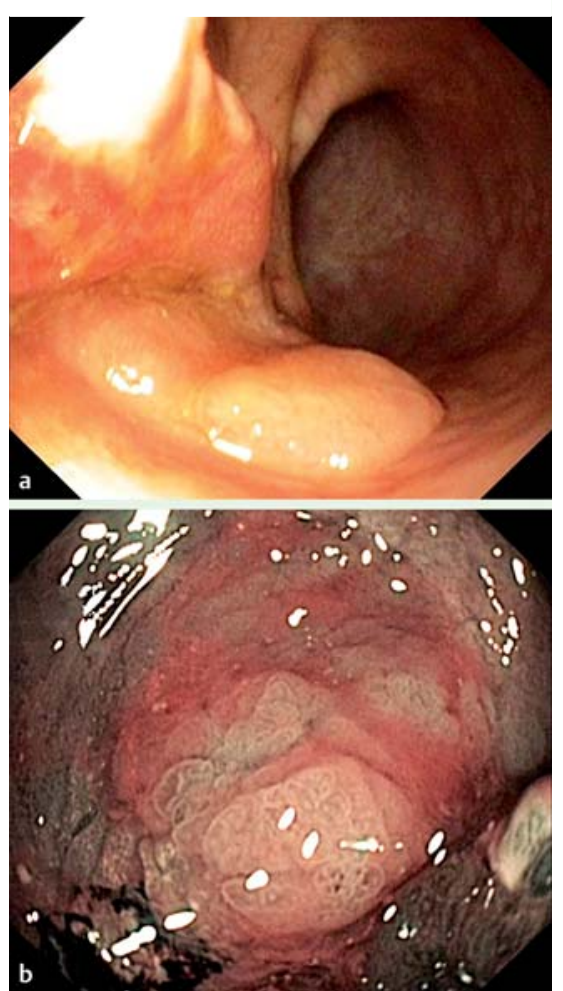

Fig. 1 a Large irregular polyp in the sigmoid colon in a 52-year-old woman with 20-year history of ulcerative colitis. $\mathbf{b}$ Note the discrete surface irregularities analyzed by narrow band imaging.

Patients with longstanding inflammatory bowel disease (IBD) have an increased risk of colorectal cancer. A causal link between chronic inflammation and cancer is well recognized. Precursor lesions include flat dysplasia (intraepithelial neoplasia) and elevated dysplasia, also known as dysplasia-associated lesion or mass (DALM) [1].

A 52-year-old woman with 20-year history of ulcerative colitis underwent surveillance colonoscopy, which disclosed a large irregular polyp in the sigmoid colon ( $\bullet$ Fig. 1). Biopsies showed a nondysplastic polyp with marked crypt dilatation and serration ( Fig.2a,b). This polyp was completely removed and a second lesion clearly showing dysplastic glands was discovered at the rectosigmoid junction, and was diagnosed as high grade DALM ( $\bullet$ Fig. 2c,d). Molecular analysis of

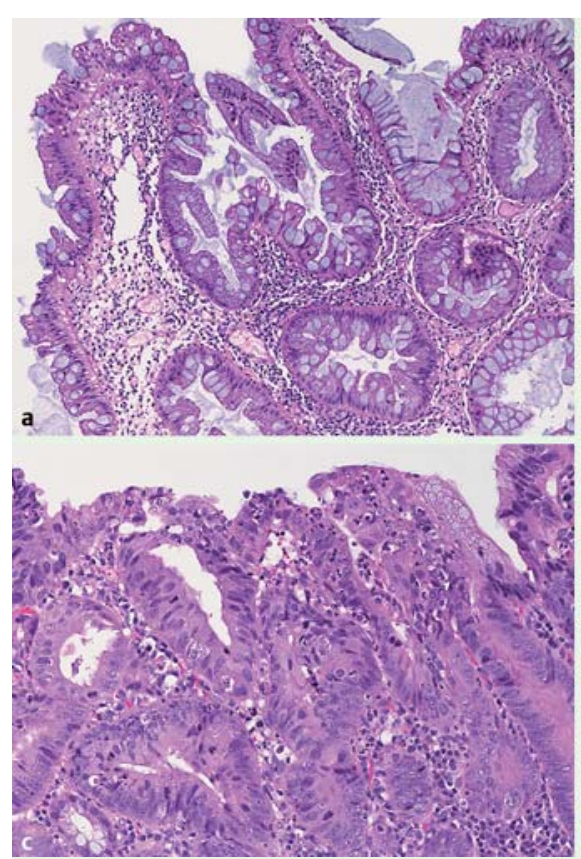

Fig. 2 a, b Nondysplastic serrated polyp with marked crypt dilatation within the sigmoid colon (hematoxylin and eosin, original $\times 100$ ). c, d Con-

the serrated polyp revealed KRAS mutation in exon 13 ( Fig.3); tests for BRAF mutation and microsatellite instability were negative.

In 2008, Srivastava et al. [2] reported a series of three patients with longstanding IBD who developed numerous "hyperplastic/serrated" colonic polyps similar to those described in the "hyperplastic/serrated" polyposis syndrome. Two patients had synchronous colorectal cancer. KRAS mutation was detected in five of the 11 polyps. These findings suggested the possibility of a serrated pathway of carcinogenesis in IBD. In the sporadic setting, sessile serrated adenomas/polyps (SSA/P) are known precursors of mainly right-sided microsatellite instable cancers. They may also be regarded as indicator lesions, as these polyps have been associated with increased risk of synchronous and/or metachronous cancer growth, particularly of the proximal colon $[3,4]$.

We believe our case to be the first description of a solitary serrated polyp with KRAS mutation, similar to the lesions occurring

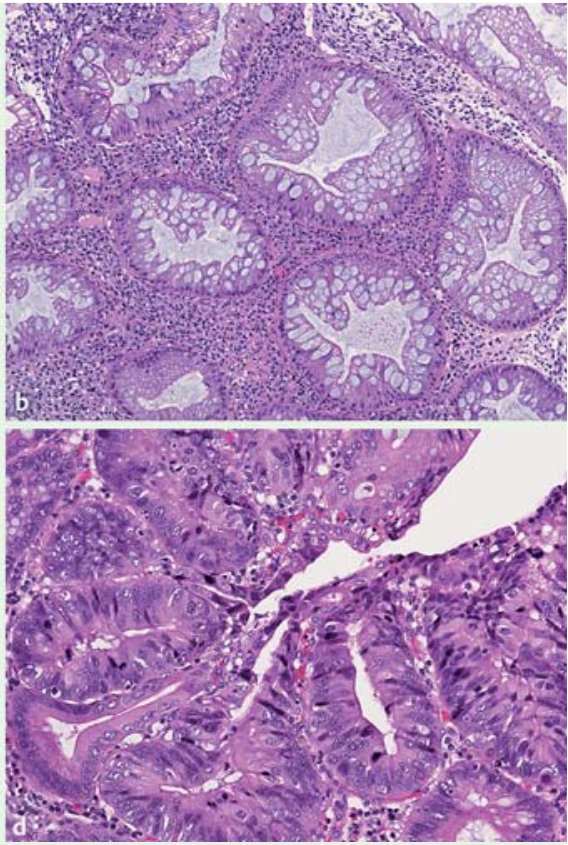

ventional high grade dysplasia-associated lesion or mass (DALM) at the rectosigmoid junction (hematoxylin and eosin, original $\times 200$ ).

as polyposis in longstanding IBD described by Srivastava et al. [2]. These nondysplastic lesions may indicate increased risk of synchronous and/or metachronous advanced neoplasia and may be the equivalent of conventional DALMs with respect to cancer prediction ("nondysplastic DALM").

Endoscopy_UCTN_Code_CCL_1AD_2AB

Competing interests: None

\section{Setaffy ${ }^{1}$, C. Högenauer ${ }^{2}$, M. Lemmerer ${ }^{3}$, C. Langner $^{1}$}

${ }^{1}$ Institute of Pathology, Medical University, Graz, Austria

${ }^{2}$ Department of Internal Medicine, Division of Gastroenterology and Hepatology, Medical University, Graz, Austria ${ }^{3}$ Department of Surgery, Krankenhaus der Barmherzigen Brüder, Academic Teaching Hospital, Graz, Austria 


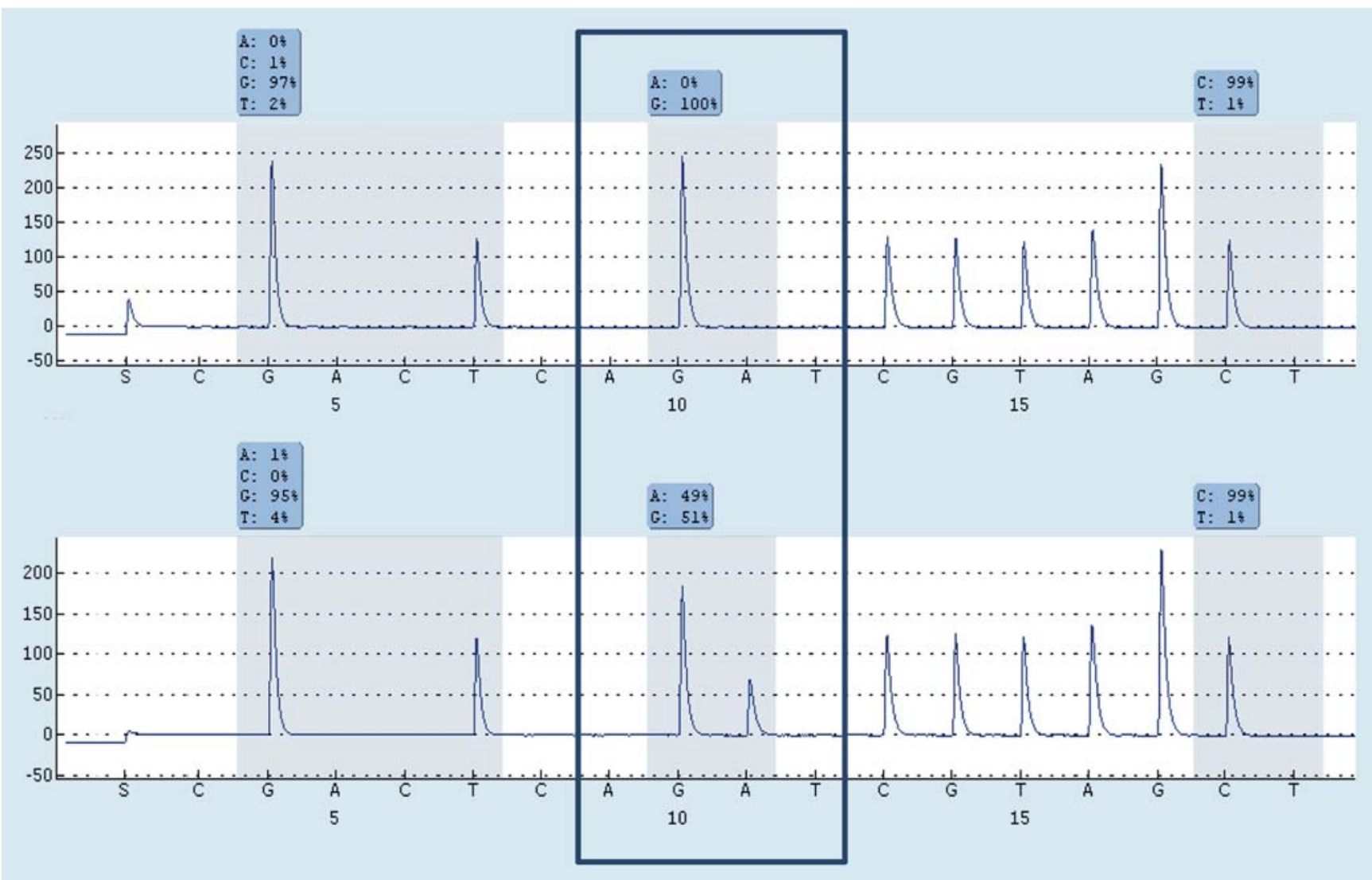

Fig. 3 Molecular analysis (pyrosequencing of the KRAS gene) of the serrated polyp showing a somatic missense mutation in codon 13: wildtype control (upper panel) and serrated polyp (lower panel).

\section{References}

1 Van Assche G, Dignass A, Bokemeyer B et al. Second european evidence-based consensus on the diagnosis and management of ulcerative colitis part 3: special situations. J Crohns Colitis 2013; 7: 1-33

2 Srivastava A, Redston M, Farraye FA et al. Hyperplastic/serrated polyposis in inflammatory bowel disease: a case series of a previously undescribed entity. Am J Surg Pathol 2008; 32: 296-303

3 Li D, Jin C, McCulloch C et al. Association of large serrated polyps with synchronous advanced colorectal neoplasia. Am J Gastroenterol 2009; 104: 695-702
4 Hiraoka S, Kato J, Fujiki S et al. The presence of large serrated polyps increases risk for colorectal cancer. Gastroenterology 2010; 139: $1503-1510$

\section{Bibliography}

DOI http://dx.doi.org/

10.1055/s-0033-1344321

Endoscopy 2013; 45: E235-E236

(c) Georg Thieme Verlag KG

Stuttgart · New York

ISSN 0013-726X

\section{Corresponding author}

\section{Langner}

Institute of Pathology

Medical University Graz

Auenbruggerplatz 25

A-8036 Graz

Austria

Fax: +43-316-38513432

cord.langner@medunigraz.at 